\title{
Optimizing electricity distribution using two-stage integer recourse models
}

\author{
Willem K. Klein Haneveld Maarten H. van der Vlerk * \\ Department of Econometrics \& OR \\ University of Groningen \\ PO Box 800, 9700 AV Groningen \\ The Netherlands
}

May 17, 2000

\begin{abstract}
We consider two planning problems faced by an electricity distributor. Electricity can be obtained both from power plants and small generators such as hospitals and greenhouses, whereas the future demand for electricity is uncertain. The price of electricity obtained from the power plants depends on quota that are to be determined in a yearly contract, whereas the (given) contracts with small generators contain various constraints on switching them on or off.
\end{abstract}

\section{Introduction}

A distributor of electricity can be seen as a middleman between suppliers and consumers of electrical power, whose aim is to satisfy demand at minimal costs. In this paper demand for electricity is aggregated, whereas a crucial distinction is made between two categories of suppliers: on the one hand there are power plants (aggregated) and in addition we consider so-called small generators. Typical examples of small generators are:

- Hospitals or other institutions with emergency generators, which can supply to the net in normal circumstances;

- Greenhouses, which produce electrical power as a by-product of generating heat;

- Industrial consumers which may be switched off for short periods of time. The net effect is the same as generating an amount of electrical power equal to their demand during such a period.

We consider two planning problems faced by a typical (Dutch) distributor of electricity (in the sequel simply called 'the distributor'). First, every year a contract with the power plants has to be negotiated. The main issue in this contract is to determine quota for the capacity of the power supply. The quota define capacity ranges for supply at any moment during the contract year, and to each range corresponds a certain price per kWh (kilo Watt hour) of supply. For a number of ranges (low, medium, and high volume of supply) such quota have to be specified at given costs per $\mathrm{kW}$ (kilo Watt) of reserved capacity. These quota are then fixed for the entire year covered by the contract, thus determining the costs

\footnotetext{
*The research of the second author has been made possible by a fellowship of the Royal Netherlands Academy of Arts and Sciences.
} 
of supply by the power plants at every moment in that year. The decision on quota is of course complicated by the uncertainty of future demand, but also by the possibility to use supply by small generators (subject to given contracts).

Once the quota are fixed, the second problem is to determine a supply schedule (that is, a schedule of supplies obtained from both power plants and small generators for every hour or even every 5 minutes) for each single day. Such a schedule has to be specified one day in advance. At this time the demand pattern for the next day is known, but only probabilistic information is available on demand in the rest of the contract year. Moreover, such a schedule has to take into account bounds on the total number of hours that a small generator can be used during the contract year.

Small generators can either supply at full capacity or not at all. Thus, the contracts with small generators specify a fixed price for a fixed capacity per time unit and usually also costs for switching them on or off. Moreover, there may be bounds on the number of time periods a small generator can be used, the number of times it can be switched on, up and down times, etc. A natural way to model these contracts is to use binary variables.

The uncertainty about future demand is modeled by random variables with known distributions. Because discrete variables are needed to model the contracts with small generators, the models we consider are stochastic programs with mixed-integer variables. In fact, as will be explained in Sections 2.3 and 3, it is very natural to model both problems as recourse models. We will argue that it is also natural to aggregate the future in each model (consisting of an entire year or the remainder of the year, respectively) and consider it as one time stage, so that both models have a two-stage integer recourse structure. For a survey on properties and general algorithms for such models we refer to [9]. In addition, several applications can be found in [13] or the bibliography [14].

We are not aware of existing literature on this specific problem. However, it is similar to the well-known unit commitment problem, which received a lot of research attention in stochastic programming (see e.g. [4] and references given there). Moreover, deregulation and the introduction of competition in the electric energy industry opens a host of research opportunities as surveyed in a recent paper in OR/MS Today [3].

The aim of this paper is twofold. First, it illustrates the modeling process for a real-life application, resulting in two-stage integer recourse models. Such models are very hard to solve in general, and - like in deterministic (mixed-)integer programming - it appears to be worthwhile to develop tailor-made solution methods for specific applications. Motivated by the structure of the models for our application, we investigate the use of valid inequalities and Lagrange relaxation. Unfortunately, we are not able to test our solution techniques on real data, since for the time being the electricity distributors we are in touch with have to focus their attention on strategic issues related to liberalization of the electricity market.

\section{Contract with power plants}

For reasons that are beyond the scope of this paper, the unit price paid by the distributor for electrical power supplied by the Dutch power plants depends on quota. For a given hour $t$, let this amount of supply be $s_{t}$. Then

$$
s_{t}=x_{t}^{1}+x_{t}^{2}+x_{t}^{3}+x_{t}^{4}
$$

with

$$
\begin{aligned}
& 0 \leq x_{t}^{1} \leq L, \\
& 0 \leq x_{t}^{2} \leq M, \\
& 0 \leq x_{t}^{3} \leq H, \\
& 0 \leq x_{t}^{4}
\end{aligned}
$$

That is, the total supply consists of four components, the first three of which are bounded from above by their respective quota $L$ (for Low), $M$ (Middle), and $H$ (High). The corresponding unit prices $c^{i}$ are such that $c^{1}<c^{2}<c^{3} \ll c^{4}$, for example $c^{1}=.04, c^{2}=.05$, 
$c^{3}=.09$, and $c^{4}=1.25 .{ }^{1}$ Because of this relation between the prices it is obvious that, for $i=2,3,4, x_{t}^{i}>0$ only if $x_{t}^{i-1}$ equals its upper bound. The interpretation is that a marginal unit of supply $s_{t}$ becomes more expensive, depending on the classification of the total supply as 'low', 'middle', 'high', or even 'very high'. Supply is considered to be very high if $s_{t}>L+M+H$; the corresponding marginal unit price $c^{4}$ reflects its function as a penalty price.

Clearly, the values of the quota $L, M$, and $H$ are very important to the distributor, since they determine the costs of the supply by the power plants for every time period. The values of the quota are set in a yearly contract between the distributor and the (collective) power plants, and remain fixed for the entire year covered by this contract. The unit costs (per kW) for reserving capacity are decreasing from $L$ to $H$, for example 260 for $L, 205$ for $M$, and 125 for $H$. Thus, when deciding on the contract with the power plants, the distributor would like to determine quota that result in the lowest total expected costs possible. These costs consist of immediate costs for reserving capacity, and expected future costs for satisfying uncertain future demand during the entire year covered by the contract. This decision under uncertainty is complicated further by the possibility to satisfy a part of the future demand by means of supply from small generators.

\subsection{Contracts with small generators}

Typically, there are about 30 to 40 small generators that can supply to the distributor. In our models the contracts with the small generators are given. In this section we discuss the nature of these contracts, that is, the price structure of supply and the various constraints that may be specified.

As stated in the Introduction, a small generator $\mathrm{SG}^{j}$ can either supply at a fixed capacity or be switched off. Hence, the contract contains a fixed price $q^{j}$ for a fixed amount of supply during one time period (say one hour) and also costs $r^{j}$ for switching the small generator on or off. Actually, we only model costs for switching on $\mathrm{SG}^{j}$ : observing that $\mathrm{SG}^{j}$ has to be switched off once it has been switched on, we take these costs equal to the sum of both costs.

The decision to use the supply of $\mathrm{SG}^{j}$ during the time period $[t, t+1), t \in\{0,1, \ldots, 23\}=$ : $T$, is modeled by a binary variable $y_{t}^{j}$, which has value one if the supply is used. If $\mathrm{SG}^{j}$ was off in the previous period $[t-1, t)$ it has to switched on, which is modeled by the binary variable $z_{t}^{j}$ which is then set to one. (It is not necessary to define binary variables for switching on, since $z_{t}^{j}=\max \left\{y_{t}^{j}-y_{t-1}^{j}, 0\right\}$ which is automatically either zero or one. However, it turns out that using binary variables $z_{t}^{j}$ allows a better formulation of the models, see Section 2.4.1.)

The contract may contain various constraints which restrict the use of $\mathrm{SG}^{j}$ per day. Below we give an overview, together with their possible mathematical formulation.

(i) Supply is not possible during certain hours of the day:

$$
\sum_{t \in T_{0}} y_{t}^{j}=0
$$

where $T_{0}$ is the corresponding set of hours.

(ii) $\mathrm{SG}^{j}$ has to be on at least $m_{1}$ and at most $M_{1}$ hours:

$$
m_{1} \leq \sum_{t \in T} y_{t}^{j} \leq M_{1}
$$

\footnotetext{
${ }^{1}$ These numbers are fictitious but the proportions are not unrealistic. The same is true for the values of other cost parameters in the sequel of this paper.
} 
(iii) $\mathrm{SG}^{j}$ has to be switched on at least $m_{2}$ and at most $M_{2}$ times:

$$
m_{2} \leq \sum_{t \in T} z_{t}^{j} \leq M_{2} .
$$

(iv) If $\mathrm{SG}^{j}$ is switched on, it has to stay on for at least $m_{3}$ and at most $M_{3}$ periods: for all $t \in T$ (with appropriate modifications for large $t$ ),

$$
\begin{array}{r}
\sum_{s=t}^{t+m_{3}-1} y_{s}^{j}-m_{3} z_{t}^{j} \geq 0 \\
\sum_{s=t}^{t+M_{3}} y_{s}^{j}-M_{3} \leq 0
\end{array}
$$

Below we use the compact notation $\left(y^{j}, z^{j}\right) \in C^{j}$ to denote these daily constraints in the contract with $\mathrm{SG}^{j}$; here $y^{j}$ is the vector $\left(y_{0}^{j}, \ldots, y_{23}^{j}\right)$ and $z^{j}$ is defined analogously. Of course, a specific contract need not contain all of the constraints mentioned above.

In addition to the daily constraints as described above, there are usually upper and lower bounds on the use of $\mathrm{SG}^{j}$ during the entire contract year. In the model for the contract with the power plants they are ignored or approximated by constraints of type (ii). They will be modeled explicitly in our second model for optimizing the daily supply schedules.

\subsection{Modeling uncertain demand for electricity}

The decision on the quota $(L, M, H)$ for next year has to be taken in advance, that is, under uncertainty about the demand for electricity. Not only the daily volume of demand is relevant, but also its dispersion over that day. We assume that a probability distribution for such demand data is available, for example based on historical data.

We aggregate the entire year covered by the contract to one period, so that the decision model becomes a two-stage model. Accordingly, and in order to arrive at a manageable model size, we do not consider random demand for each future day separately, but instead consider so-called representative days. An example of a representative day is a working day in the spring season, which represents all working days in that season. This aggregation is motivated by the assumption that all working days in the spring have a similar demand pattern and volume. On the other hand, the demand is different from that on e.g. a holiday or a Saturday in the same season, and also different from the pattern and/or volume observed on working days during other seasons.

Let $R^{1}, \ldots, R^{N}$ denote the collection of representative days, for example consisting of working days, Saturdays, Sundays, and holidays for each season, giving $N=16$. From the available data now a discrete probability distribution can be estimated for the representative days. For each $i$, its realizations $\omega^{i k}=\left(\omega_{0}^{i k}, \ldots, \omega_{23}^{i k}\right), k=1, \ldots, K^{i}$, are then possible demand patterns on representative day $R^{i}$. Of course, the corresponding probabilities $p^{i k}$ are such that $\sum_{k} p^{i k}$ equals the relative number of days of type $R^{i}$ in a year.

To simplify the notation, we will use a single index for $\omega$ and $p$ from now on. 


\subsection{Optimizing the contract with the power plants}

For given quota $(L, M, H)$ and a realization of a one-day demand pattern $\omega$, the minimal costs for satisfying this demand are

$$
\begin{aligned}
& v(L, M, H, \omega)=\min _{x, y, z} \sum_{t \in T}\left(\sum_{i=1}^{4} c^{i} x_{t}^{i}+\sum_{j \in J}\left(q^{j} y_{t}^{j}+r^{j} z_{t}^{j}\right)\right) \\
& \text { s.t. } \quad \sum_{i=1}^{4} x_{t}^{i}+\sum_{j \in J} b^{j} y_{t}^{j} \geq \omega_{t}, \quad t \in T \\
& x_{t}^{1} \leq L, x_{t}^{2} \leq M, x_{t}^{3} \leq H, \quad t \in T \\
& \left(y^{j}, z^{j}\right) \in C^{j}, \quad j \in J \\
& x_{t}^{i} \geq 0, \quad i=1, \ldots, 4, t \in T \\
& y_{t}^{j}, z_{t}^{j} \in\{0,1\}, \quad j \in J, t \in T
\end{aligned}
$$

where $J$ is the set of small generators. The first constraint reflects that demand has to be satisfied at all times, either by supply $x_{t}^{i}$ from the power plants or by supply from small generators, which have fixed capacity $b^{j}, j \in J$. This is a mixed-integer (binary) optimization problem, with $4 \cdot|T|$ continuous variables and $2 \cdot|J| \cdot|T|$ binary variables. (Notation: $|S|$ denotes the cardinality of a set $S$.)

Thus, for fixed quota $(L, M, H)$, the expected costs for satisfying the demand of one day are $\mathbb{E}_{\omega}[v(L, M, H, \omega)]$, giving yearly expected costs $365 \cdot \mathbb{E}_{\omega}[v(L, M, H, \omega)]$. In the contract with the power plants, the goal of the distributor is to determine quota that minimize the total expected costs, which is the sum of the direct cost for fixing the quota and the expected costs of satisfying demand during the contract year. Thus, the goal is to find an optimal solution of the following two-stage integer recourse problem:

$$
\begin{array}{ll}
\min _{L, M, H} & C^{1} L+C^{2} M+C^{3} H+365 \cdot \mathbb{E}_{\omega}[v(L, M, H, \omega)] \\
\text { s.t. } & L, M, H \geq 0,
\end{array}
$$

where $C^{i}, i=1,2,3$, are the unit costs for reserving capacity by means of the quota, and $v$ is the second-stage value function defined above.

In the next section we propose techniques that appear to be useful for solving this model. However, since we do not have access to realistic data, we will not be able to apply these ideas in numerical experiments. Instead, we performed a simple simulation, using artificial (but not unrealistic) data and the assumption that the small generators provide $10 \%$ of the supply needed. The results show that for optimal quota the direct costs are about $52 \%$ of the total expected costs, which are approximately 304 million Dutch guilders (Dfl). Moreover, we found that small deviations from the optimal quota (plus or minus 5\% for $L$ ) give an increase of total expected costs of up to 7 million Dfl $(2.3 \%)$. These results indicate that it is worthwhile to investigate the recourse model presented above.

\subsection{On solving the integer recourse model}

In order to solve the integer recourse model we have to be able to evaluate the objective function, or at least to provide a reasonably good approximation. That is, for given quota $(L, M, H)$ and a realization of the demand $\omega$, we have to solve the mixed-integer secondstage problem (1). Indeed, we need to perform these calculations many times, so that it is important to do this efficiently. To this end we consider two techniques, both inspired by the structure of this particular problem. Such problem-specific solution methods (both algorithms and heuristics) have proved to be very fruitful for deterministic mixed-integer problems, see e.g. [10] [2]. This case study illustrates the use of this strategy in a stochastic setting. 
Since supply obtained from small generators is more expensive than supply from the power plants at unit costs $c^{1}$, and because the demand for electricity is relatively low and stable during the night (23.00 till 7.00), it follows that it is never optimal to use supply from small generators during the night. Therefore, this part of the twenty-four hours can be ignored in our optimization model, so that from now on we only consider supply and demand for the hours $[t, t+1), t \in T:=\{7,8, \ldots, 22\}$.

Even after this reduction, the second-stage problem is a mixed-integer problem with at least 1280 binary variables, given that there are 30 to 40 small generators involved. Our first computations (using a different but correct formulation of the second-stage problem) already failed for an instance with as few as 5 small generators: the branch-and-bound solver ZOOM [12] did not find an optimal solution in several hours CPU time, see [5]. Therefore, we need to improve the formulation of the second-stage problem as shown below.

\subsubsection{Valid inequalities}

First, we observe that the constraints $\left(y^{j}, z^{j}\right) \in C^{j}, j \in J$, do not depend on the firststage decisions $(L, M, H)$ nor on the observed demand pattern $\omega$. Consequently, we can improve the formulation of the second-stage problem by adding so-called valid inequalities (see e.g. [10]), which are valid in all second-stage problems, that is, for every choice of $(L, M, H)$ and $\omega$.

Adding valid inequalities results in a better lower bound obtained by solving the LP relaxation of the problem, which hopefully improves the performance of the usual branchand-bound algorithm for mixed-integer problems. In general it is difficult to find strong valid inequalities that result in a substantial reduction of the computing time. However, since the same valid inequalities can be used for all second-stage problems, we can afford to spend (a lot of) time on determining such strong valid inequalities.

For example, assume that a small generator has to be switched on at least $m$ times per day, and that if it is switched on, it has to stay on for at least $n$ periods (see (ii) and (iv) in Section 2.1). Then the following valid inequalities prove to be very useful:

$$
\begin{aligned}
& \sum_{t \in T} y_{t} \geq m n, \\
& \sum_{s \in T \backslash S(t)} z_{s} \geq m-1 \quad \forall t \in T,
\end{aligned}
$$

where $S(t):=\{t, t+1, \ldots, t+n\}$. The first of these valid inequalities is trivial (but very powerful); the second reflects the observation that the small generator can be switched on only once in $n+1$ consecutive time periods, so that it has to be switched on at least $m-1$ times during the remaining periods.

Using these valid inequalities (among others) in the formulation (1) of the second-stage problem, the instance with 5 small generators is now solved by CPLEX 5.0 [1] in 1.02 seconds (on a Pentium $450 \mathrm{Mhz}$ with $384 \mathrm{MB}$ memory). This spectacular reduction of the computing time is partly due to defining the variables $z_{t}^{j}$ to be binary: this allows CPLEX to automatically generate additional valid inequalities (so-called cover and clique inequalities). In any case, this illustrates that valid inequalities (user defined or automatically generated) work very well for this problem.

\subsubsection{Lagrange relaxation}

Even with valid inequalities included, the second-stage problem (1) can not yet be solved fast enough. Indeed, instead of 5 small generators as in the computational example, the actual number of small generators is 30 to 40 . Moreover, the second-stage problem needs to be solved many times, so that even a computing time of 1 second is prohibitive. Next we consider further reduction of computing time by applying Lagrange relaxation. 
In the second-stage problem (1), the small generators are loosely coupled in the sense that they are only connected through the constraints prescribing that demand should be satisfied at all times:

$$
\sum_{i=1}^{4} x_{t}^{i}+\sum_{j \in J} b^{j} y_{t}^{j} \geq \omega_{t}, \quad t \in T .
$$

It is therefore natural to consider Lagrange relaxation of these constraints, since this results in a separable second-stage problem. Indeed, for $\lambda \in \mathbb{R}_{+}^{|T|}$, the Lagrange function is

$$
\begin{aligned}
\Lambda(\lambda, L, M, H, \omega)= & \min _{x} \sum_{T} \sum_{i=1}^{4}\left(c^{i}-\lambda_{t}\right) x_{t}^{i} \\
& \text { s.t. } \quad x \in X(L, M, H) \\
& +\sum_{J} \min _{y^{j}, z^{j}} \sum_{T}\left(\left(q^{j}-\lambda_{t} b^{j}\right) y_{t}^{j}+r^{j} z_{t}^{j}\right) \\
\text { s.t. } & \left(y^{j}, z^{j}\right) \in C^{j} \\
& +\sum_{T} \lambda_{t} \omega_{t},
\end{aligned}
$$

where $X(L, M, H, \omega):=\left\{x \geq 0: x_{t}^{1} \leq L, x_{t}^{2} \leq M, x_{t}^{3} \leq H \quad \forall t \in T\right\}$.

The first term of (3) is a simple LP problem for which the the optimal value can be given in closed form. Obviously, since $x_{t}^{4}$ is not bounded from above, the optimal value is $-\infty$ if $\lambda_{t}>c^{4}$ for any $t \in T$, so that optimal values of the Lagrange multipliers $\lambda$ are contained in the hypercube $\left[0, c^{4}\right]^{|T|}$. Given the interpretation of $\lambda_{t}$ as the unit price of electricity obtained from an alternative supplier, the upper bound $c^{4}$ is natural, since an unlimited amount of electricity can be obtained from the power plants at unit $\operatorname{costs} c^{4}$.

The second term of the Lagrange function (3) consists of $|J|$ separate pure-binary problems: one for each individual small generator. Using valid inequalities as discussed in the previous section, each of these small problems is solved by CPLEX in 0.01 seconds on average. Consequently, each evaluation of the Lagrange function for given $\lambda, L, M, H$ and $\omega$, takes about 0.5 seconds.

The Lagrange dual

$$
\max _{\lambda \in\left[0, c^{4}\right]^{|T|}} \Lambda(\lambda, L, M, H, \omega)
$$

provides a lower bound for $v(L, M, H, \omega)$. This convex non-smooth problem can be solved efficiently by special purpose software, for example by NOA (see Kiwiel [6]). The interpretation of $\lambda$ suggests that $\lambda_{t} \in\left[c^{2}, c^{3}\right], t \in T$, is a good starting point for such iterative methods.

\subsubsection{Implementation}

The goal of using valid inequalities and Lagrange relaxation is to speed up the evaluation of the second-stage value function defined in (1). Of course, this is only one ingredient of an algorithm for solving the two-stage integer recourse problem (2), resulting in optimal quota $L, M$, and $H$. Since we do not have the necessary data, we did not attempt such computations. However, it seems that existing algorithms such as Stochastic Branch-andBound [11] can be applied.

\section{Supply schedule for the next day}

The second problem faced by the distributor is to determine a schedule for the next day, specifying the amount of supply from the power plants and the small generators for each 
period during that day. This schedule has to be conveyed to the suppliers one day ahead (at noon).

The goal of the distributor is to find a schedule that minimizes the costs of supply for the next day, and that also takes into account bounds on the total number of hours that a small generator can be used during the whole year.

The following information is available:

(i) The current quota $(L, M, H)$ and corresponding unit prices in the contract with the power plants.

(ii) The contracts with the small generators, including restrictions on the total usage per year.

(iii) An accurate prediction of the electricity demand for the next day, and probabilistic information on demand during the remainder of the year.

In practice, the prediction of demand for the next day is accurate enough to be treated as deterministic information, denoted by $d_{t}, t \in T$. Hence, also this problem can be formulated as a two-stage integer recourse model:

$$
\begin{aligned}
& \min _{x, y, z} \sum_{t \in T}\left(\sum_{i=1}^{4} c^{i} x_{t}^{i}+\sum_{j \in J}\left(q^{j} y_{t}^{j}+r^{j} z_{t}^{j}\right)\right)+Q(y) \\
& \text { s.t. } \quad \sum_{i=1}^{4} x_{t}^{i}+\sum_{j \in J} b^{j} y_{t}^{j} \geq d_{t}, \quad t \in T \\
& x_{t}^{1} \leq L, x_{t}^{2} \leq M, x_{t}^{3} \leq H, \quad t \in T \\
& \left(y^{j}, z^{j}\right) \in C^{j}, \quad j \in J \\
& x_{t}^{i} \geq 0, \quad i=1, \ldots, 4, t \in T \\
& y_{t}^{j}, z_{t}^{j} \in\{0,1\}, \quad j \in J, t \in T,
\end{aligned}
$$

where the function $Q$ reflects the expected recourse costs due to not meeting the restrictions on the yearly usage of the small generators. Below we propose a simple recourse structure to model these future costs.

The first stage of the current model is exactly the same as the second-stage part of the previous model. Thus, the valid inequalities that we derived for the constraints $\left(y^{j}, z^{j}\right) \in$ $C^{j}, j \in J$, can again be used to speed up the computations. Moreover, the same Lagrange relaxation as above results here in a completely separated problem, since the separation of the first stage matches the separation of the function $Q$ which is due to the simple recourse structure. Before we discuss this in more detail, we first present the simple recourse model, starting with the corresponding representation of uncertainty about future demand.

\subsection{Future demand for supply from small generators}

The simple recourse model that we have in mind assigns penalty costs to a surplus or shortage of yearly usage for each small generator. To model this, we need to translate the known distribution of future demand for electricity to a distribution of future demand for supply from the small generators.

Consider again a collection of representative days $R^{1}, \ldots, R^{N}$, as defined in Section 2.2. For each $R^{i}$, with its typical demand patterns, we compute the optimal supply schedules giving in particular the optimal usage $h^{i j}$ of each small generator $\mathrm{SG}^{j}, j \in J$. On day $\tau$ of the year, let the random vector $\eta_{\tau}=\left(\eta_{\tau}^{1}, \ldots, \eta_{\tau}^{N}\right)$ denote the remainder of the year, with $\eta_{\tau}^{i}$ the number of days of type $i$ (obviously, $\sum_{i=1}^{N} \eta_{\tau}^{i}=365-\tau$ ). Given the probabilities of the realizations of $\eta_{\tau}$, we can compute the distribution of the number 
of hours $\xi_{\tau}^{j}$ that supply from $\mathrm{SG}^{j}$ is needed in the remainder of the year, since

$$
\xi_{\tau}^{j}=\sum_{i=1}^{N} h^{i j} \eta_{\tau}^{i}
$$

By this preprocessing step we can obtain the distribution of the random vector $\xi_{\tau}=$ $\left(\xi_{\tau}^{1}, \ldots, \xi_{\tau}^{|J|}\right)$ for each day $\tau$.

\subsection{Modeling expected penalty costs}

Let $L^{j}$ and $U^{j}$ be the lower and upper bound on the total number of hours that $\mathrm{SG}^{j}$ can be used during the contract year. At day $\tau$ we know how much supply is taken from $\mathrm{SG}^{j}$ so far, giving current bounds

$$
\begin{aligned}
& L_{\tau}^{j}:=L^{j}-\sum_{s=1}^{\tau-1} u_{s}^{j} \\
& U_{\tau}^{j}:=U^{j}-\sum_{s=1}^{\tau-1} u_{s}^{j},
\end{aligned}
$$

where $u_{s}^{j}$ is the number of hours that $\mathrm{SG}^{j}$ was used on day $s$. Thus, for given usage $u_{\tau}=\left(u_{\tau}^{1}, \ldots, u_{\tau}^{|J|}\right)$ and a realization $\xi_{\tau}$ of desired future supply by the small generators, the value function

$$
\begin{array}{cl}
v\left(u_{\tau}, \xi_{\tau}\right):=\min & q^{+} y^{+}+q^{-} y^{-} \\
\text {s.t. } & y^{+} \geq \xi_{\tau}+u_{\tau}-U_{\tau} \\
& y^{-} \geq L_{\tau}-\left(\xi_{\tau}+u_{\tau}\right) \\
& y^{+}, y^{-} \in \mathbb{R}_{+}^{|J|}
\end{array}
$$

gives the minimal penalty costs for not meeting the yearly lower and upper bounds for each of the small generators. Since any surplus can be bought at unit costs $c^{4}$ from the power plants, we take all components of $q^{+}$equal to $c^{4}$. On the other hand, if the total yearly supply from $\mathrm{SG}^{j}$ falls below the lower bound $L^{j}$, it seems reasonable to charge unit penalty costs equal to a fraction of $q^{j}+r^{j}$.

Due to the simple recourse structure, this second-stage problem is separable. Hence, the function $v$ gives the total penalty costs as the sum of the penalty costs for each small generator individually:

$$
v\left(u_{\tau}, \xi_{\tau}\right)=\sum_{j=1}^{|J|} v^{j}\left(u_{\tau}^{j}, \xi_{\tau}^{j}\right),
$$

where $v^{j}$ is the one-dimensional version of (5). Consequently, also the expected penalty costs function $Q\left(u_{\tau}\right):=\mathbb{E}_{\xi_{\tau}}\left[v\left(u_{\tau}, \xi_{\tau}\right)\right]$ is separable, with terms $Q^{j}\left(u_{\tau}^{j}\right)=\mathbb{E}_{\xi_{\tau}^{j}}\left[v^{j}\left(u_{\tau}^{j}, \xi_{\tau}^{j}\right)\right]$.

Using this penalty costs model may lead to the undesirable result that already early in the year the supply obtained from one or more small generators is close to one of its bounds, thus limiting the options for scheduling in the rest of the year. (Even if such a sequence of supply schedules has minimal total expected costs, it is undesirable in the sense that it does not correspond to current practice.) This effect can be avoided by means of the following refinement of the second-stage problem. The idea is to 'aim' total expected supply from each $\mathrm{SG}^{j}$ at the average value $\left(L_{\tau}^{j}+U_{\tau}^{j}\right) / 2$, which is achieved by putting a small penalty 
on deviations from this value. In the refined model, penalty costs are given by

$$
\begin{aligned}
& w\left(u_{\tau}, \xi_{\tau}\right)=\min \quad q^{1+} y^{1+}+q^{2+} y^{2+}+q^{1-} y^{1-}+q^{2-} y^{2-} \\
& \text { s.t. } \quad y^{1+}+y^{2+}-y^{1-}-y^{2-}=\xi_{\tau}+u_{\tau}-\left(L_{\tau}+U_{\tau}\right) / 2 \\
& y^{1+} \leq\left(U_{\tau}-L_{\tau}\right) / 2 \\
& y^{1-} \leq\left(U_{\tau}-L_{\tau}\right) / 2 \\
& y^{1+}, y^{2+}, y^{1-}, y^{2-} \in \mathbb{R}_{+}^{|J|},
\end{aligned}
$$

where $q^{2+}$ and $q^{2-}$ are equal to $q^{+}$and $q^{-}$in the previous model, and the values of $q^{1+}<q^{2+}$ and $q^{1-}<q^{2-}$ are for example determined by numerical experiments (and are possibly decreasing with $\tau$ ).

This refined model is called a multiple simple recourse model (see [7]), and has the same separability properties as the simple recourse model. In fact, it follows from Theorem 3.1 in [8] that such a multiple simple recourse model is equivalent to a simple recourse model in the following sense.

Corollary 3.1 Consider the one-dimensional multiple simple recourse value function $w$,

$$
\begin{aligned}
w(s):=\min & q^{2+} y^{2+}+q^{1+} y^{1+}+q^{1-} y^{1-}+q^{2-} y^{2-} \\
\text { s.t. } \quad & y^{1+}+y^{2+}-y^{1-}-y^{2-}=s \\
& y^{1+} \leq u \\
& y^{1-} \leq l \\
& y^{1+}, y^{2+}, y^{1-}, y^{2-} \in \mathbb{R}_{+}
\end{aligned}
$$

and the one-dimensional simple recourse value function $v$,

$$
\begin{aligned}
v(s):=\min & q^{2+} y^{+}+q^{2-} y^{-} \\
\text {s.t. } & y^{+}-y^{-}=s \\
& y^{+}, y^{-} \in \mathbb{R}_{+},
\end{aligned}
$$

where $q^{2+}>q^{1+}>0>-q^{1-}>-q^{2-}$.

For any random variable $\chi$ with finite mean value, whose cumulative distribution function (cdf) is denoted by $F$,

$$
\mathbb{E}_{\chi}[w(\chi-x)]=\mathbb{E}_{\psi}[v(\psi-x)]-c, \quad x \in \mathbb{R},
$$

where $\psi$ is a random variable with $c d f W$,

$$
W(s)=\frac{q^{1+}+q^{1-}}{q^{2+}+q^{2-}} F(s)+\frac{q^{2+}-q^{1+}}{q^{2+}+q^{2-}} F(s+u)+\frac{q^{2-}-q^{1-}}{q^{2+}+q^{2-}} F(s-l),
$$

The constant $c$ is given by

$$
c=\frac{q^{2+}\left(q^{2-}-q^{1-}\right) l+q^{2-}\left(q^{2+}-q^{1+}\right) u}{q^{2+}+q^{2-}} .
$$

PROOF. The result follows from Theorem 3.1 in [8] by straightforward computation.

Since the multiple simple recourse expected value function (EVF) $\mathbb{E}_{\xi}[w(\xi-x)], x \in \mathbb{R}^{n}$, is separable, Corollary 3.1 implies that this function is equal (up to a known constant) to a related simple recourse EVF with known distribution of the right-hand side parameters. Hence, such a multiple simple recourse model can be solved by existing algorithms for simple recourse models. 


\subsection{Optimal supply schedules}

Putting the ingredients discussed above together, we see that the problem of finding an optimal supply schedule for the next day can be modeled as a two-stage (multiple) simple recourse model, with mixed-binary first-stage variables and continuous second-stage variables.

In practice, next days supply schedule has to be specified for each quarter of an hour or even every 5 minutes. In the latter case there are 11520 binary first-stage variables (to model 30 small generators). Even using valid inequalities as discussed in Section 2.4.1, direct computations for the full model are probably too time consuming. Also for this model Lagrange relaxation of the constraints relating supply and demand appears a useful option, since the resulting problem separates in $|J|$ (multiple) simple recourse problems for each $\mathrm{SG}^{j}$ individually (plus an LP problem corresponding to supply from the power plants).

Again, we did not actually perform these computations because the necessary data were not available to us.

\section{Conclusions}

We have shown that the application at hand can (or even should) be modeled by twostage mixed-integer recourse models. This illustrates the wide applicability of such models. Moreover, we have investigated the use of problem specific solution techniques, thus following an approach that has been very successful for deterministic mixed-integer problems. In particular, we showed how the use of valid inequalities and Lagrange relaxation may lead to computationally tractable models for problems of realistic size.

In future research we will investigate similar approaches to other specific mixed-integer recourse problems.

\section{References}

[1] Using the CPLEX Callable Library. CPLEX Optimization Inc., 1995.

[2] M. Dell'Amico, F. Maffioli, and S. Martello, editors. Annotated bibliographies in combinatorial optimization. Wiley, 1997.

[3] L.F. Escudero and M. Pereira. Power play goals. OR/MS Today, 27(2):42-46, 2000.

[4] N. Gröwe-Kuska, K.C. Kiwiel, M.P Nowak, W. Römisch, and I. Wegner. Power management in a hydro-thermal system under uncertainty by Lagrangian relaxation. Stochastic Programming E-Print Series, available at http://dochost.rz . huberlin.de/speps/, 2000.

[5] C. Kamerling. Een model voor de inzet-planning van decentrale opwekkers, 1997. Master thesis, Department of Econometrics, University of Groningen, The Netherlands.

[6] K.C. Kiwiel. Proximity control in bundle methods for convex nondifferentiable minimization. Mathematical Programming, $46(1$ (Ser. A)):105-122, 1990.

[7] W.K. Klein Haneveld. Duality in stochastic linear and dynamic programming, volume 274 of Lecture Notes in Economics and Mathematical Systems. Springer-Verlag, Berlin, 1986.

[8] W.K. Klein Haneveld, L. Stougie, and M.H. van der Vlerk. On the convex hull of the simple integer recourse objective function. Annals of Operations Research, 56:209224, 1995. 
[9] W.K. Klein Haneveld and M.H. van der Vlerk. Stochastic integer programming: General models and algorithms. Annals of Operations Research, 85:39-57, 1999.

[10] G.L. Nemhauser and L.A. Wolsey. Integer and Combinatorial Optimization. Wiley, New York, 1988.

[11] V.I. Norkin, G.Ch. Pflug, and A. Ruszczyński. A branch and bound method for stochastic global optimization. Mathematical programming, 83(3):425-450, 1998.

[12] J. Singhal, R. E. Marsten, and T. L. Morin. Fixed order branch-and-bound methods for mixed-integer programming: The ZOOM system. ORSA Journal on Computing, 1:44-51, 1989.

[13] L. Stougie and M.H. van der Vlerk. Stochastic integer programming. In M. Dell'Amico, F. Maffioli, and S. Martello, editors, Annotated Bibliographies in Combinatorial Optimization, chapter 9, pages 127-141. Wiley, 1997.

[14] M.H. van der Vlerk. Stochastic programming bibliography. World Wide Web, http: //mally.eco.rug.nl/biblio/stoprog.html, 1996-2000. 